\title{
AVALIAÇÃO DA PRODUTIVIDADE DOS CENTROS DE ESPECIALIDADES ODONTOLÓGICAS DO ESTADO DE PERNAMBUCO NOS ANOS DE 2015 E 2016
}

\author{
K. F. dos S MARTINS ${ }^{1}$, C. A. S. CEISSLER ${ }^{2}$, P. H. P. de M. VAZ ${ }^{1}$, N. FIGUEIREDO ${ }^{2}$ \\ ${ }^{1}$ Universidade Federal de Pernambuco, Programa de Pós-graduação em Gestão e Economia \\ da Saúde \\ ${ }^{2}$ Universidade de Federal de Pernambuco, Programa de Pós-graduação em Saúde \\ Coletiva \\ E-mail para contato: kadidja_martins@hotmail.com
}

\begin{abstract}
RESUMO - O presente estudo de abordagem quantitativa de caráter avaliativo normativo, foi desenvolvido a partir de dados secundários disponíveis no Sistema de Informação Ambulatorial do Sistema Único de Saúde (SIA-SUS). Analisou-se o desempenho dos Centros de Especialidades Odontológicas (CEO) tipo II do estado de Pernambuco nos anos de 2015 e 2016 através do indicador Cumprimento Global de Metas (CGM). Após a avaliação, o desempenho dos estabelecimentos foi classificado em: péssimo, ruim, bom e ótimo. No ano de 2015, para as especialidades de Endodontia, Cirurgia Oral e Periodontia a maioria dos CEO não cumpriu a meta. Em 2016, 52,2\% cumpriram a meta de Periodontia e 91,3\% a de Procedimentos Básicos. Em 2015 o desempenho ruim foi a predominante (43,5\%), enquanto que em 2016 foi o regular $(34,8 \%)$. Nesse sentido, observa-se que os CEO apresentam dificuldades para a garantia do quantitativo mínimo de procedimentos especializados, excetuando-se os Procedimentos Básicos.
\end{abstract}

Palavras-chaves: Avaliação em Saúde. Atenção Secundária à Saúde. Saúde Bucal.

ABSTRACT - The present study of quantitative approach of normative evaluative character, was developed from secondary data available in the Outpatient Information System of the Unified Health System (SIA-SUS). The performance of the Type II Dental Specialties Centers (CEO) of the state of Pernambuco in the years 2015 and 2016 was analyzed through the Global Goal Compliance (CGM) indicator. After the evaluation, the performance of the establishments was classified in: bad, bad, good and great. In the year 2015, for the specialties of Endodontics, Oral Surgery and Periodontics most of the CEO did not fulfill the goal. In 2016, 52.2\% met the goal of Periodontics and $91.3 \%$ of Basic Procedures. In 2015 the poor performance was the predominant one (43.5\%), while in 2016 it was the regular one (34.8\%). In this sense, it is observed that CEOs present difficulties in guaranteeing the minimum quantity of specialized procedures, except for the Basic Procedures.

Keywords: Health Evaluation. Secondary Care. Oral Health. 


\section{INTRODUÇÃO}

A Política Nacional de Saúde Bucal (PNSB) - Brasil Sorridente lançada em 2004, teve na implantação de Centros de Especialidades Odontológicas (CEO) como forma de ampliar o acesso a serviços odontológicos especializados (BRASIL, 2004). Os CEO são estabelecimentos de referência para atenção básica, ofertando no mínimo os serviços de diagnóstico bucal, com ênfase no diagnóstico e detecção do câncer bucal, periodontia especializada, cirurgia oral menor dos tecidos moles e duros, endodontia e atendimento a portadores de necessidades especiais (BRASIL, 2006). Apesar do avanço quantitativo, faz-se necessária a avaliação destes estabelecimentos, em prol da melhoria contínua do seu desempenho.

Os CEO podem ser classificados como CEO do tipo I (com três cadeiras odontológicas), Tipo II (com quatro a seis cadeiras) ou Tipo III (com sete ou mais cadeiras). De acordo com o tipo, os CEO recebem um incentivo financeiro do Ministério da Saúde para implantação, custeio mensal e um valor adicional para adesão à Rede de Cuidado à Pessoa com Deficiência (BRASIL, 2006; BRASIL, 2012; BRASIL, 2017). O repasse financeiro é condicionado a uma produção mínima mensal estabelecida na portaria GM/MS n ${ }^{\circ} 1464$ de 24 de junho de 2011, a qual indica que a meta estabelecida para os CEO tipo II são 110 procedimentos básicos exclusivos para portadores de necessidades especiais, 90 de periodontia, 60 de endodontia e 90 de cirurgia oral (BRASIL, 2011). Nesse contexto, o presente trabalho objetiva avaliar o cumprimento global dessas metas de produtividade dos CEO tipo II do estado de Pernambuco nos anos de 2015 e 2016.

\section{MATERIAIS E MÉTODOS}

Realizou-se um estudo quantitativo de caráter avaliativo normativo desenvolvido a partir de dados secundários disponíveis no Sistema de Informação Ambulatorial do Sistema Único de Saúde (SIA/SU). Examinou-se a produtividade de todos os CEO tipo II do estado de Pernambuco registrados no Cadastro Nacional de Estabelecimentos de Saúde (CNES) nos anos de 2015 e 2016, com base nos valores indicados na Portaria $\mathrm{n}^{\mathrm{o}}$ 1.464/2001. A alocação dos procedimentos registrados foi realizada de acordo com os subgrupos de especialidade: procedimentos básicos, periodontia, endodontia e cirurgia oral. Em seguida, calculou-se o indicador denominado Cumprimento Global de Metas (CGM) (GOES et al., 2012). Este é resultante do quociente da média mensal de procedimentos realizados de cada subgrupo de especialidades odontológicas pelo número de procedimentos correspondente à meta deste subgrupo multiplicado por 100 . Considerou-se que os serviços que cumpriram percentual igual ou superior a $100 \%$ da meta normatizada para cada subgrupo teve sua meta atingida. Mediante essa metodologia, o desempenho dos serviços foi classificado como: péssimo (CEO que não cumpriu nenhuma meta); ruim (1 meta); regular (2 metas); bom (3 metas); e, ótimo (4 metas).

\section{RESULTADOS E DISCUSSÃO}

Foram identificados 23 CEO do tipo II em Pernambuco, distribuídos em 18 municípios, dos quais $14 \mathrm{com}$ mais de 100 mil habitantes. A maior parte possuía Índice de Desenvolvimento Humano (IDH) menor que 0,7 $(n=17)$ e cobertura de saúde bucal menor que $50 \%(n=12)$. Conforme a tabela 1 , no ano de 2015 a meta de produtividade foi alcançada apenas para procedimentos básicos (91,3\%), já em 2016 a meta foi alcançada 
para periodontia $(52,2 \%)$ e procedimentos básicos (91,3\%). No ano de 2016 as especialidades de endodontia e cirurgia oral apresentaram aumento no da meta em relação a 2015 , passando de $8,7 \%$ para $21,7 \%$ em endodontia, e de $21 \%$ para $39,1 \%$ em cirurgia oral. Observou-se que a maior parte $(43,5 \%)$ dos estabelecimentos teve desempenho ruim em 2015, ao passo que a maioria $(34,8 \%)$ teve desempenho regular em 2016 (tabela 2).

Tabela - Avaliação do Cumprimento da Produção Mínima Estabelecida por especialidade para CEO Tipo II, Pernambuco 2015-2016.

\begin{tabular}{ccrlrl}
\hline \multirow{2}{*}{ Especialidade } & Cumprimento da Produção & \multicolumn{2}{c}{$\mathbf{2 0 1 5}$} & \multicolumn{2}{c}{$\mathbf{2 0 1 6}$} \\
\cline { 3 - 6 } & Mínima Estabelecida & $\mathbf{n}$ & $\mathbf{\%}$ & $\mathbf{n}$ & $\mathbf{\%}$ \\
\hline \multirow{2}{*}{ Endodontia } & Sim & 2 & $8,7 \%$ & 5 & $21,7 \%$ \\
& Não & 21 & $91,3 \%$ & 18 & $78,3 \%$ \\
\multirow{2}{*}{ Cirurgia Oral } & Sim & 5 & $21 \%$ & 9 & $39,1 \%$ \\
& Não & 18 & $78 \%$ & 14 & $60,9 \%$ \\
Procedimentos Básicos & Sim & 17 & $73,9 \%$ & 21 & $91,3 \%$ \\
& Não & 6 & $26,1 \%$ & 2 & $8,7 \%$ \\
Periodontia & Sim & 6 & $26,1 \%$ & 12 & $52,2 \%$ \\
& Não & 17 & $73,9 \%$ & 11 & $47,8 \%$ \\
\hline
\end{tabular}

Tabela - Avaliação do desempenho da produtividade de CEO Tipo II, Pernambuco 2015-2016.

\begin{tabular}{crlrll}
\hline \multirow{2}{*}{ Desempenho } & \multicolumn{2}{c}{$\mathbf{2 0 1 5}$} & \multicolumn{2}{c}{$\mathbf{2 0 1 6}$} \\
\cline { 2 - 6 } & $\mathbf{n}$ & $\mathbf{\%}$ & $\mathbf{n}$ & $\mathbf{\%}$ \\
\hline Péssimo & 6 & $26,1 \%$ & 2 & $8,7 \%$ \\
Ruim & 10 & $43,5 \%$ & 6 & $26,1 \%$ \\
Regular & 2 & $8,7 \%$ & 8 & $34,8 \%$ \\
Bom & 4 & $17,4 \%$ & 3 & $13 \%$ \\
Ótimo & 1 & $4,3 \%$ & 4 & $17,4 \%$ \\
Total & $\mathbf{2 3}$ & $\mathbf{1 0 0 \%}$ & $\mathbf{2 3}$ & $\mathbf{1 0 0 \%}$ \\
\hline
\end{tabular}

No Brasil, em 2007, elaborou-se uma modelo de avaliação de desempenho para o CEO pela medição do cumprimento global de metas segundo macrorregião. Os resultado mostraram um desempenho ruim na maioria dos CEO, com exceção do Centro-Oeste e Sudeste, em que a maioria teve bom desempenho (GOES et al., 2012). Em Pernambuco, 2009, um estudo com CEOs revelou que 40,9\% dos centros analisados apresentaram bom desempenho, em contrapartida $31,8 \%$ tiveram desempenho ruim. Com o maior cumprimento de metas no subgrupo de atenção básica e o menor no de cirurgia oral menor. O estudo enfatiza a necessidade de uma adequação dos critérios e normas para implantação e monitoramento dos serviços (FIGUEIREDO, 2009).

\section{CONCLUSÃO}

Nesse sentido, observa-se que os CEOs apresentam dificuldades para a garantia do quantitativo mínimo de procedimentos especializados, excetuando-se os Procedimentos Básicos. Destacando, desta maneira, a importância da gestão dos centros de avaliar e monitorar os serviços, para que supere os déficits na produtividade. 


\section{REFERÊNCIAS}

BRASIL. Ministério da Saúde. Diretrizes da política nacional de saúde bucal. Brasília: Ministério da Saúde; 2004.

BRASIL. [acesso 04 jun. 2017] Disponível em: http://dados.gov.br/dataset/mspainelsage 30/resource/3c909609-b10e-454d-8d6c$\underline{\mathrm{d} 03297306891}$

BRASIL. Ministério da Saúde. Portaria no 1341, de 13 de junho de 2012. Define os valores dos incentivos de implantação e de custeio mensal dos Centros de Especialidades Odontológicas - CEO e dá outras providências. Diário Oficial da União, Brasilia, pag. 74, 02 julho 2012. Seção 1 .

BRASIL. Ministério da Saúde. Portaria n 1464, de 24 de junho de 2011. Altera o Anexo da Portaria no 600/GM/MS, de 23 de março de 2006, que institui o financiamento dos Centros de Especialidades Odontológicas(CEO). Diário Oficial da União, Brasilia, pag. 112, 27 junho 2011. Seção 1.

BRASIL. Ministério da Saúde. Portaria no 599/GM, de 23 de março de 2006. Define a implantação de Centros de Especialidades Odontológicas (CEOs) e de Laboratórios Regionais de Próteses Dentárias (LRPDs) e estabelece critérios, normas e requisitos para seu credenciamento. Diário Oficial da União, Brasília, pag 51. 24 mar. 2006. Seção 1.

FIGUEIREDO, N.; GOES, P. S. A.. Construção da atenção secundária em saúde bucal: um estudo sobre os Centros de Especialidades Odontológicas em Pernambuco, Brasil. Cad. Saúde Pública, Rio de Janeiro, v. 25, n. 2, p. 259-267, Fevereiro 2009.

GOES, P. S. A et al . Avaliação da atenção secundária em saúde bucal: uma investigação nos centros de especialidades do Brasil. Cad. Saúde Pública, Rio de Janeiro, v. 28, supl. p. s81-s89, Janeiro 2012 . 